\title{
Qualidade de aplicativos móveis sobre prevenção e controle da sífilis
}

\author{
Quality of mobile apps for syphilis prevention and control \\ Calidad de las aplicaciones móviles de prevención y control de la sífilis
}

Nathanael de Souza Maciel ${ }^{1}$ (B)

Diego da Silva Ferreira² (D)

Vitória Talya dos Santos Sousa ${ }^{1}$ (1)

Hévila Ferreira Gomes Medeiros Braga ${ }^{1}$ (1)

Gutemberg dos Santos Chaves $^{1}$ (D)

Leilane Barbosa de Sousa ${ }^{1}$ (c)

1. Universidade da Integração Internacional da Lusofonia Afro-Brasileira, Instituto de Ciências da Saúde. Redenção, CE, Brasil.

2. Universidade Estadual do Ceará. Fortaleza, $\mathrm{CE}$, Brasil.
Autor correspondente:

Nathanael de Souza Maciel.

E-mail: nathanael.souza.inf@gmail.com

Recebido em 07/05/2021.

Aprovado em 07/09/2021.

\section{Resumo}

Objetivo: Avaliar a qualidade dos aplicativos móveis sobre prevenção e controle da sífilis. Métodos: Estudo descritivo e avaliativo dos aplicativos para dispositivos móveis em ambientes virtuais. A busca foi operacionalizada no período de janeiro a fevereiro de 2020, nas plataformas Android e IOS. Foi definida a questão norteadora: "Qual a qualidade dos aplicativos móveis sobre prevenção e controle da sífilis disponíveis em lojas virtuais?". As palavras-chaves "Sífilis", "Syphilis" e "prevenção da sífilis" foram utilizadas como estratégia de pesquisa nas lojas. Os aplicativos foram avaliados por três autores, por meio da Mobile App Rating Scale, voltada para avaliação da qualidade de aplicativos móveis em saúde. Os dados foram apresentados em quadros sinópticos e tabelas. Resultados: Dez aplicativos foram incluídos, todos disponíveis gratuitamente somente para o sistema operacional Android. A média geral dos aplicativos foi de 2,8, não atingindo a pontuação mínima de aceitabilidade de 3,0. 0 engajamento dos aplicativos foi avaliado com média 2,2, funcionalidade com 3,7, estética com 2,8 e as informações contidas nos aplicativos com média 2,6. Conclusão e implicações para a prática: Evidenciou-se que a qualidade dos aplicativos sobre sífilis deve ser melhorada. Urge a necessidade de avaliação da eficácia dessas intervenções para prevenção e controle da sífilis.

Palavras-chave: Educação em Saúde; Infecções Sexualmente Transmissíveis; Prevenção de Doenças; Sífilis; Tecnologias da Informação.

\section{Abstract}

Objective: To evaluate the quality of mobile apps on syphilis prevention and control. Methods: A descriptive and evaluative study of mobile apps in virtual environments. The search was operationalized from January to February 2020, on Android and IOS platforms. The following guiding question was defined: "What is the quality of mobile apps on syphilis prevention and control available in virtual stores?"The keywords "Syphilis", "Sifilis" and "syphilis prevention" were used as a search strategy in the stores. The apps were evaluated by three authors, through the Mobile App Rating Scale, aimed at assessing the quality of mobile health apps. The data were presented in synoptic charts and tables. Results: Ten apps were included, all available free of charge for the Android operating system. The overall mean score of the apps was 2.8 , not reaching the minimum acceptability score of 3.0 . App engagement was evaluated with a mean of 2.2, functionality with 3.7, aesthetics with 2.8 and information contained in apps with a mean of 2.6. Conclusion and implications for the practice: It was evidenced that the quality of apps on syphilis should be improved. There is an urgent need to evaluate the effectiveness of these interventions for syphilis prevention and control.

Keywords: Education in Health; Sexually Transmitted Infections; Disease Prevention; Syphilis; Information Technology.

\section{Resumen}

Objetivo: Evaluar la calidad de las aplicaciones móviles en materia de prevención y control de la sífilis. Métodos: Estudio descriptivo y evaluativo de aplicaciones móviles en ámbitos virtuales. La búsqueda se llevó a cabo de enero a febrero de 2020 , en plataformas Android e IOS. Se definió la pregunta orientadora: “¿Qué calidad tienen las aplicaciones móviles sobre prevención y control de la sífilis, disponibles en las tiendas online?". Las palabras clave "Sífilis", "Syphilis "y "prevención de la sífilis" se utilizaron como estrategia de investigación en las tiendas. Las aplicaciones fueron evaluadas por tres autores a través de la Escala de Clasificación de Aplicaciones Móviles, destinada a evaluar la calidad de las aplicaciones móviles de salud. Los datos se presentaron en tablas y cuadros sinópticos. Resultados: Se incluyeron diez aplicaciones, todas disponibles de forma gratuita solo para el sistema operativo Android. El promedio general de las solicitudes fue de 2,8 , sin alcanzar la puntuación mínima de aceptabilidad de 3,0. La participación en aplicaciones se evaluó con una media de 2,2, funcionalidad con 3,7, estética con 2,8 e información contenida en aplicaciones con una media de 2,6. Conclusión e implicaciones para la práctica: Se evidencia que debe mejorarse la calidad de las aplicaciones sobre la sífilis. Es urgente evaluar la eficacia de estas intervenciones para la prevención y el control de la sífilis.

Palabras-clave: Educación en Salud; Infecciones Sexualmente Transmitidas; Prevención de Enfermedades; Sífilis; Tecnología de la Información 


\section{INTRODUÇÃO}

O uso de dispositivos móveis tem crescido no cenário mundial. Até o final de 2019, 5,2 bilhões de pessoas possuíam serviços móveis, representando $67 \%$ da população global. Estima-se que esse número alcançará um total de 5,8 bilhões até 2025 , representando $70 \%$ da população mundial ${ }^{1}$.

Diante desse contexto, a mHealth ou saúde móvel, definida como prática médica e de saúde pública apoiada por dispositivos móveis, como telefones celulares ${ }^{2}$, pode ser particularmente útil no fornecimento de intervenções em saúde, visto que permite realizar ações em escala, cobrir áreas geográficas amplas e fornecer tecnologias de maneiras altamente personalizadas, com base nas preferências ou características dos usuários ${ }^{3}$.

De fato, a $\mathrm{mHeal}$ th está rapidamente entrando nos ambientes institucionais de assistência à saúde e nos espaços privados, cercada por uma promessa de revolucionar a assistência à saúde, aumentando o autogerenciamento e o empoderamento dos pacientes, promovendo a eficiência, a prevenção de doenças e também a acessibilidade à saúde em todo o mundo 4 .

Os canais de comunicação digital, como aplicativos, oferecem ampla cobertura, permitem que as mensagens sejam direcionadas a grupos ou indivíduos específicos, oferecem potencial para melhorar a entrega de informações e suporte nos domínios de saúde sexual e reprodutiva ${ }^{5}$. Assim, os aplicativos podem fornecer intervenções de prevenção de infecções sexualmente transmissíveis (IST) ${ }^{6}$, dentre as quais, a sífilis.

Para a sífilis, em 2016, a estimativa global de prevalência em homens e mulheres foi de $0,5 \%$, com valores regionais variando de 0,1 a 1,6\%, correspondendo a 19,9 milhões de casos. Em relação à incidência global de sífilis, estima-se 1,7 casos por 1.000 mulheres e 1,6 por 1000 homens, traduzindo 6,3 milhões de casos em mulheres e homens com idades entre 15 e 49 anos em $2016^{7}$.

Nesse sentido, a estratégia global sobre IST estabelece a meta de reduzir a incidência de sífilis em $90 \%$ e reduzir a incidência de sífilis congênita para $<50$ casos por 100.000 nascidos vivos até $2030^{\circ}$. Todavia, os índices e tendências na prevalência e na incidência de sífilis adquirida e congênita sugerem aumento de $\operatorname{casos}^{9,10}$.

Diante desse panorama, considerando a crescente utilização de dispositivos móveis como promissoras intervenções em saúde e a ascensão das taxas de sífilis, justifica-se o interesse no desenvolvimento deste estudo, cujos resultados permitirão conhecer a qualidade dos aplicativos sobre prevenção e controle da sífilis, verificando se são ferramentas com potencial para promover resultados positivos sobre o cuidado na prevenção e controle da sífilis pela população em geral.

Portanto, objetivou-se avaliar a qualidade dos aplicativos móveis sobre prevenção e controle da sífilis.

\section{MÉTODOS}

Estudo descritivo e avaliativo, operacionalizado por meio da busca de aplicativos para dispositivos móveis nos principais ambientes virtuais, no período de janeiro a fevereiro de 2020 . Foram utilizados dois smartphones para a busca em dois sistemas operacionais distintos: um Moto G5SPlus, para a pesquisa na Play Store (Android, Google) e um Iphone 8, para as buscas na Apple Store (iOS, Apple).

Foi definida a questão norteadora: "Qual a qualidade dos aplicativos móveis sobre prevenção e controle da sífilis disponíveis em lojas virtuais?". Para responder a esta pergunta, as palavraschaves "Sífilis", "Syphilis" e "prevenção da sífilis" foram utilizadas como estratégia de pesquisa nas lojas virtuais de aplicativos.

Foram incluídos os aplicativos móveis que abordassem a prevenção e o controle da sífilis. Foram excluídos: aplicativos duplicados; que se destinavam a profissionais e a serviços de saúde; em idiomas diferentes do inglês, português e espanhol; que abordassem outras IST; sobre eventos; que fossem apenas meio de divulgação ou de exposição digital de revistas ou periódicos; e aplicativos que necessitassem de acesso com login específico ou institucional.

Seguindo os critérios deste estudo, os aplicativos foram avaliados por meio de um instrumento validado denominado Mobile App Rating Scale (MARS) ${ }^{11}$. A escolha dessa ferramenta se deu por ser uma medida multidimensional confiável, simples e objetiva para testar, classificar e avaliar a qualidade de aplicativos móveis em saúde ${ }^{11}$.

O MARS contém 23 itens em 3 seções: classificação, qualidade do aplicativo e satisfação. Neste estudo, a satisfação (qualidade subjetiva) não foi avaliada, uma vez que essa seção não era considerada no escore médio de qualidade do aplicativo, devido à sua natureza subjetiva ${ }^{11}$. Cada item do MARS usa uma escala de 5 pontos ( 1 - inadequado; 2 - ruim; 3-aceitável; 4 - bom; 5 - excelente). A seção de classificação é utilizada apenas para fins descritivos, contendo variáveis como nome do aplicativo avaliado, sistema operacional, versão do aplicativo e data da última atualização. A seção de qualidade de aplicativo possui 19 itens e classifica os aplicativos em quatro subescalas: engajamento, funcionalidade, estética e qualidade da informação. O MARS é pontuado calculando as pontuações médias das subescalas de qualidade do aplicativo e a pontuação média total. Existe uma seção de qualidade subjetiva, contendo 4 itens que avaliam a satisfação total do usuário, classificados separadamente como itens individuais. O MARS demonstrou excelente desempenho interno, consistência $(a=0,92)$ e confiabilidade entre avaliadores $(\mathrm{ICC}=0,85)^{11}$. Além desses dados, também foram consideradas as avaliações públicas disponibilizadas nas plataformas de download dos aplicativos, realizadas pelos usuários individualmente. Essa avaliação é baseada no número proporcional de avaliações, variando de uma a cinco estrelas, conforme a qualidade atual do aplicativo.

Cada aplicativo foi utilizado e avaliado por três autores deste estudo, visando assegurar a consistência e a precisão dos resultados do processo de mensuração. A nota de cada critério foi uma média das notas. Os dados foram armazenados no software Microsoft Office Excel e apresentados em quadros sinópticos e tabelas. 


\section{RESULTADOS}

As pesquisas efetivadas nos dois sistemas operacionais identificaram 511 aplicativos. Excluindo-se 61 aplicativos duplicados, havia 450 aplicativos. No entanto, 52 eram em idiomas diferentes do inglês, português e espanhol e 369 apresentavam outras temáticas. Dos 29 aplicativos restantes, 08 aplicativos foram excluídos por serem destinados aos profissionais da saúde; 07 abordavam outras IST; 02 precisavam de login para acesso e 2 eram aplicativos de revista ou evento científico. Ao fim, 10 aplicativos foram incluídos. A Figura 1 apresenta o fluxograma de seleção dos aplicativos.

Todos os aplicativos incluídos estavam disponíveis gratuitamente para o sistema operacional Android, mas nenhum para a plataforma IOS. Os dados sobre a caracterização desses aplicativos são apresentados no Quadro 1.
O principal idioma dos aplicativos era o inglês. A maioria possuía classificação livre. A Tabela 1 apresenta a pontuação em subescala e geral de aplicativos classificados com MARS. Não foi possível classificar o item 19, que fornece uma medida da base de evidências para os aplicativos, pois, nos resultados do presente estudo, os aplicativos encontrados não contemplavam esse item. Contudo, isso não alterou o cálculo da média do escore.

O aplicativo "Sexually Transmitted Disease and Infections" teve a maior média de pontuação total de MARS (média 3,4). As próximas mais altas foram "Sexo Seguro" $(3,3)$ e "Sexually Transmitted Diseases Free" $(3,2)$. O "Syphilis: Cure And Tips" obteve a pontuação mais baixa $(2,3)$. A média de todos os aplicativos foi de 2,8 , não atingindo a pontuação mínima de aceitabilidade de 3,0 .

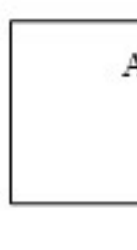 na Apple Store $(\mathrm{n}=11)$

licativos identificados

na Play Store

$(n=500)$
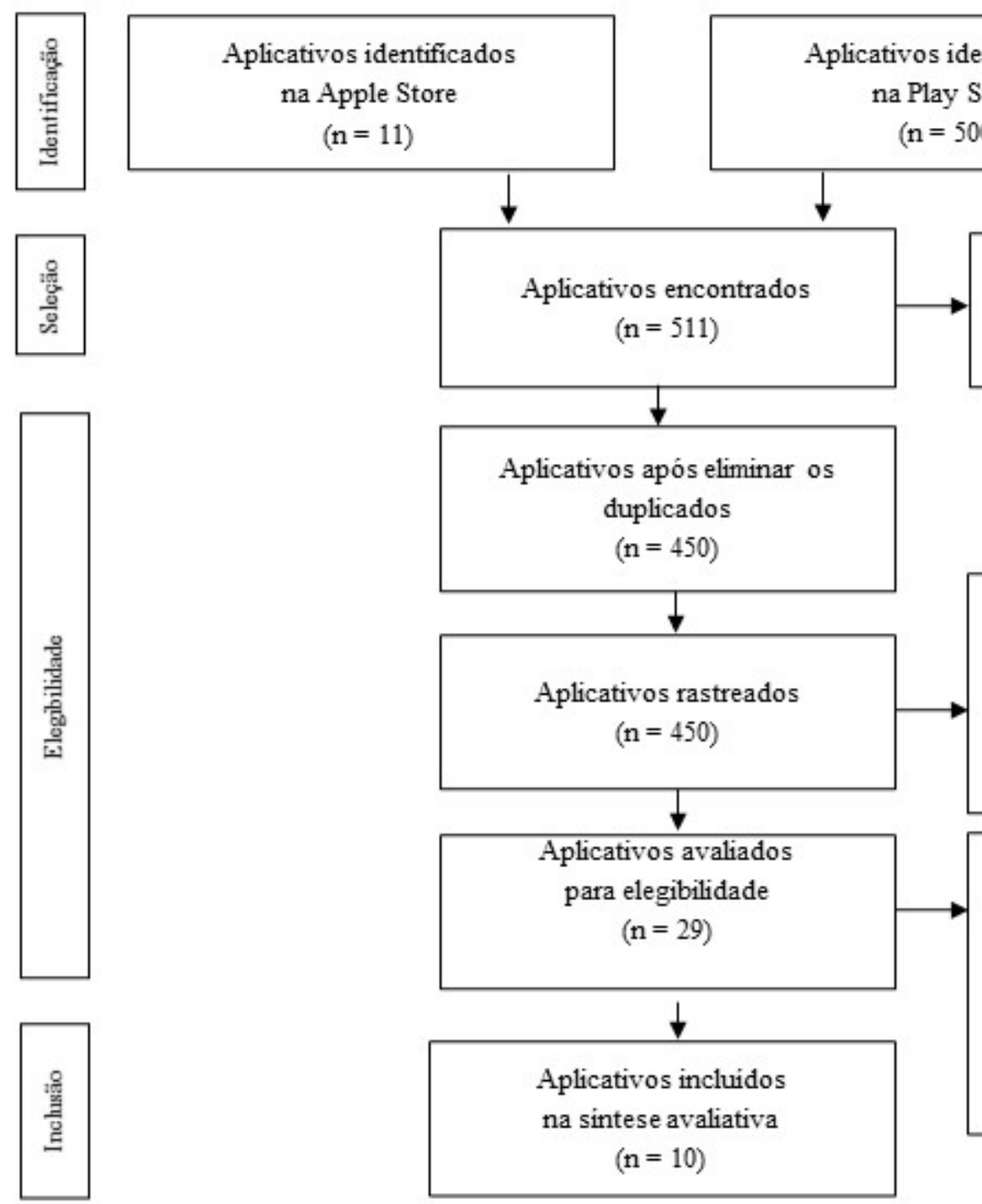

曼

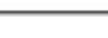

$\longrightarrow \begin{gathered}\text { Aplicativos excluidos com } \\ \text { motivos ( } \mathrm{n}=19) \\ \text { Para profissionais ( } \mathrm{n}=8) \\ \text { Não responde à pergunta }(\mathrm{n}=7) \\ \text { Precisa de login }(\mathrm{n}=2) \\ \text { Divulgação revista }(\mathrm{n}=2)\end{gathered}$

Figura 1. Fluxograma de identificação do processo de seleção dos aplicativos. Fonte: Dados da pesquisa.
Aplicativos excluidos com motivos $(\mathrm{n}=421)$

Outro idioma $(\mathrm{n}=52$ Não responde à pergunta $(\mathrm{n}=369)$

Aplicativos excluidos com motivos $(\mathrm{n}=19)$ $(\mathrm{n}=61)$ 
Quadro 1. Caracterização dos aplicativos incluídos.

\begin{tabular}{|c|c|c|c|c|c|c|}
\hline Aplicativo* & Desenvolvedor & Idioma & Download & $\begin{array}{c}\text { Nota/No de } \\
\text { avaliações }\end{array}$ & $\begin{array}{c}\text { Data de } \\
\text { atualização }\end{array}$ & Classificação \\
\hline $\begin{array}{l}\text { 1. How to Treat } \\
\text { Syphilis }\end{array}$ & NonitaDev & Inglês & $>1000$ & $0 / 0$ & $25 / 06 / 2018$ & Livre \\
\hline $\begin{array}{l}\text { 2. Sexually } \\
\text { Transmitted } \\
\text { Diseases Free }\end{array}$ & Medical 360 & Inglês & $>50$ & $0 / 0$ & 21/08/2019 & Livre \\
\hline $\begin{array}{l}\text { 3. Sexually } \\
\text { Transmitted } \\
\text { Disease and } \\
\text { Infections }\end{array}$ & Sonal Hadiya & 15 idiomas & $>1000$ & $0 / 0$ & 01/04/2019 & Livre \\
\hline $\begin{array}{l}\text { 4. Syphilis and } \\
\text { Treatment }\end{array}$ & Fumo & Inglês & $>1$ & $0 / 0$ & $19 / 01 / 2020$ & 14 \\
\hline 5. Sexo Seguro & Eworld Tech & Português & $>500$ & $0 / 0$ & 21/06/2019 & Livre \\
\hline 6. Syphilis Info & Programming Is Fun & Inglês & $>100$ & $0 / 0$ & 05/11/2019 & 12 \\
\hline $\begin{array}{l}\text { 7. Sexually } \\
\text { transmitted } \\
\text { diseases }\end{array}$ & JACOAPPS & Inglês & $>1000$ & $4,4 / 7$ & 03/01/2020 & Livre \\
\hline $\begin{array}{l}\text { 8. Sexually } \\
\text { Transmitted } \\
\text { Diseases }\end{array}$ & Education_free & Inglês & $>1000$ & $4,7 / 7$ & $14 / 10 / 2018$ & Livre \\
\hline $\begin{array}{l}\text { 9. Syphilis: Cure } \\
\text { And Tips }\end{array}$ & Biemultimedia & Inglês & $>100$ & $0 / 0$ & 09/01/2020 & Livre \\
\hline $\begin{array}{l}\text { 10. STDs } \\
\text { Sexually } \\
\text { Transmitted } \\
\text { Diseases }\end{array}$ & Dr_Apps & Inglês & $>500$ & $0 / 0$ & $16 / 10 / 2018$ & Livre \\
\hline
\end{tabular}

Fonte: Dados da pesquisa.

*As versões classificadas dos aplicativos podem não estar disponíveis nas lojas no momento da publicação, pois podem ter sido substituídas por versões mais recentes.

Na seção do engajamento, foi avaliado se os aplicativos eram divertidos, interessantes, customizáveis, interativos e bem orientados para o público. Verificou-se que os aplicativos não utilizavam todas as estratégias para o aumento do entretenimento e do interesse, por exemplo, através da gamificação $(2,4)$; não permitiam qualquer personalização, não fornecendo espaço para configurações ou preferências de usuários $(1,3)$; possuíam características não interativas ou falta de resposta à interação do utilizador, não enviando alertas, mensagens, lembretes ou feedback $(1,8)$; tinham conteúdos aceitáveis, mas não direcionados ao público-alvo $(3,0)$. Nessa seção, os aplicativos foram avaliados com média 2,2.

Na seção da funcionalidade, foram avaliados a navegação, a lógica dos fluxos, o design e a facilidade para aprender o aplicativo. Os aplicativos funcionavam em geral, mas alguns apresentaram problemas técnicos, como lentidão $(3,5)$; eram fáceis de aprender a usar ou tinham instruções claras $(4,0)$; possuíam navegação (mover entre telas) apropriada e fácil de usar $(3,8)$; possuíam interações coerentes e intuitivas em todos os componentes e telas $(3,5)$. A funcionalidade dos aplicativos foi avaliada, no geral, com média 3,7.

Na seção da estética, foram avaliados o design gráfico, o apelo visual geral, o esquema de cores e a consistência estilística. Os aplicativos possuíam layouts satisfatórios, mas com alguns problemas, como o tamanho de botões, ícones ou menu $(3,0)$; a qualidade gráfica moderada e o design visual consistente $(3,0)$. Todavia, possuíam pouco apelo visual ou mau uso de cores, tornando-os visualmente não atrativos $(2,4)$. A estética dos aplicativos foi avaliada com média 2,8.

Na seção da informação, foi avaliada a qualidade dos textos e referências, se eram de fonte credível. Os aplicativos foram considerados imprecisos em relação à sua descrição na loja, pois contêm poucas informações dos componentes e funções descritas $(2,6)$. No tocante aos objetivos e metas mensuráveis e realizáveis, especificados na loja ou dentro do próprio aplicativo, são enumeradas algumas metas, porém os aplicativos têm pouca 
Tabela 1. Escores da avaliação conforme a MARS.

\begin{tabular}{|c|c|c|c|c|c|}
\hline Aplicativo* & Engajamento & Funcionalidade & Estética & Informação $^{+}$ & Geral \\
\hline 1. How to Treat Syphilis & 2,3 & 4,3 & 3,1 & $2,4^{\ddagger}$ & 3,0 \\
\hline $\begin{array}{l}\text { 2. Sexually Transmitted } \\
\text { Diseases Free }\end{array}$ & 2,3 & 4,0 & 3,2 & 3,2 & 3,2 \\
\hline $\begin{array}{l}\text { 3. Sexually Transmitted } \\
\text { Disease and Infections }\end{array}$ & 2,7 & 3,8 & 3,8 & $3,1^{\S}$ & 3,4 \\
\hline 4. Syphilis and Treatment & 2,3 & 3,6 & 2,7 & 2,8 & 2,8 \\
\hline 5. Sexo Seguro & 2,4 & 3,9 & 3,6 & 3,3 & 3,3 \\
\hline 6. Syphilis Info & 2,5 & 3,3 & 3,0 & $2,7^{\ddagger}$ & 2,9 \\
\hline $\begin{array}{l}\text { 7. Sexually transmitted } \\
\text { diseases }\end{array}$ & 1,9 & 3,7 & 2,0 & $2,4^{\ddagger}$ & 2,5 \\
\hline $\begin{array}{l}\text { 8. Sexually Transmitted } \\
\text { Diseases }\end{array}$ & 1,9 & 3,4 & 2,4 & $2,3^{\ddagger}$ & 2,5 \\
\hline 9. Syphilis: Cure And Tips & 1,7 & 3,3 & 2,1 & 2,3 & 2,3 \\
\hline $\begin{array}{l}\text { 10. STDs Sexually } \\
\text { Transmitted Diseases }\end{array}$ & 1,9 & 3,4 & 2,3 & $2,0^{\ddagger \S}$ & 2,4 \\
\hline
\end{tabular}

Fonte: Dados da pesquisa.

*As versões classificadas dos aplicativos podem não estar disponíveis nas lojas no momento da publicação, pois podem ter sido substituídas por versões mais recentes;

${ }^{\dagger}$ o escore de qualidade da informação excluiu o item 19 do MARS;

‡o escore de qualidade da informação excluiu o item 14 do MARS, visto que a descrição e o aplicativo não listavam objetivos ou metas a serem alcançadas;

${ }^{\S}$ o escore de qualidade da informação excluiu o item 17 do MARS, porque não havia informação visual dentro do aplicativo (por exemplo, ele contém apenas áudio ou texto).

chance de alcançá-las $(2,9)$. Sobre a qualidade da informação, os aplicativos possuem conteúdo apropriado $(3,0)$ e, sobre a quantidade, as informações são adequadas e abrangentes, mas não concisas $(3,1)$. A informação visual, apresentada por meio de gráficos e imagens, foi adequada, entretanto, imprecisa, confusa ou errada $(3,0)$. Além disso, em alguns aplicativos, não há informação visual, contendo apenas texto. No que concerne à credibilidade, as fontes especificadas na loja ou dentro dos aplicativos, quando identificadas, foram consideradas questionáveis $(1,7)$. As informações contidas nos aplicativos foram avaliadas com média 2,6.

\section{DISCUSSÃO}

Os aplicativos estão sendo incorporados cada vez mais como estratégias de subsídios ao processo de ensino e aprendizagem. Todavia, poucos aplicativos sobre sífilis foram encontrados neste estudo. Isso reforça uma lacuna nas ferramentas que podem ser utilizadas para a prevenção desse agravo. Enfatizase que a inserção dessas tecnologias no aprendizado emerge como mais uma possibilidade de instruir-se ${ }^{12}$, proporcionando o autocuidado do sujeito, pois eles possuem informações relevantes para tornar o indivíduo em principal protagonista do seu contexto saúde e doença.

$\mathrm{Na}$ busca realizada, constatou-se que todos os aplicativos identificados sobre sífilis estavam disponíveis apenas para
Android e gratuitamente. Efetivamente, o desenvolvimento de aplicativos para Android é mais simples em razão do acesso mais profundo ao sistema operacional e altos níveis de personalização são possíveis, corroborando para que esse sistema operacional ocupe $87 \%$ do mercado ${ }^{13}$. Ademais, o custo também parece ser uma preocupação significativa para não usuários e usuários, visto que a maioria das pessoas não quer pagar pelos aplicativos e interrompem o uso quando descobrem ser necessário pagamento no aplicativo ${ }^{14}$.

Um dos fatores que pode dificultar o acesso aos aplicativos sobre a sífilis consiste no idioma em que o aplicativo está disponível. A maioria dos aplicativos encontrados estava no idioma inglês. $O$ uso dessa língua pode, em alguns casos, limitar o acesso às informações e ocasionar interpretações equivocadas por não nativos do idioma, tornando-se um obstáculo para as pessoas que não possuem o grau de instrução necessário para leitura e compreensão ${ }^{15}$. Nesse sentido, o desenvolvimento de aplicativos sobre sífilis na língua portuguesa se configura como uma estratégia de inclusão digital das pessoas leigas, podendo ser uma forma de democratização do acesso ao conhecimento de modo amplo e acessível, gerando impactos positivos no processo saúde-doença.

Verificou-se que os aplicativos para sífilis não possuíam todos os recursos para prender a atenção dos usuários. Em um estudo entre os usuários que fizeram o download de aplicativos de saúde e que não os usam mais, um quantitativo de $40,5 \%$ 
revelou que a descontinuidade se deu pela perda de interesse no aplicativo ${ }^{14}$. Nessa perspectiva, seria apropriado envolver os usuários finais dos aplicativos (sejam profissionais de saúde ou pacientes) no processo de projetá-los durante os estágios iniciais, a fim de identificar suas reais necessidades e características ${ }^{16}$. Dessa forma, aplicativos sobre sífilis, embasados pelo contexto de inserção do usuário, podem ter a capacidade de serem mais interessantes.

Conseguir que os usuários se envolvam com um aplicativo de saúde móvel é fundamental para o sucesso de aplicativos e para as intervenções focadas na mudança de comportamento na saúde. A incorporação de recursos mais personalizáveis em aplicativos móveis de saúde, direcionados a subgrupos importantes, pode ajudar a facilitar o uso por mais tempo e, consequentemente, gerar melhores resultados para a saúde ${ }^{17}$. Além disso, a eficácia das intervenções digitais é mediada por fatores associados ao envolvimento do usuário em intervenções e determinantes da mudança do comportamento da saúde ${ }^{12}$. Não obstante, no presente estudo, detectou-se que as características de personalização e de gamificação foram frágeis, o que pode fazer com que o usuário perca o interesse pelos aplicativos sobre sífilis.

Com o intuito de aperfeiçoar esse envolvimento, a gamificação pode ser um meio eficaz de direcionar componentes motivacionais e os jogos podem ser eficazes para desencadear o interesse nos indivíduos e aumentar a popularidade dos aplicativos ${ }^{18}$. Torna-se concludente que o jogo interativo aumenta a motivação dos jogadores para melhorar os comportamentos de saúde e o autocuidado, em uma variedade de cenários e populações clínicas, podendo atrair e manter a atenção, sendo um componente chave para uma mudança efetiva de comportamento ${ }^{19}$.

Para o desenvolvimento de um aplicativo, é imprescindível que ele possua algumas características: disponibilização de informações relevantes, fidedignas e atuais; linguagem compreensível; imagens e disposições das informações visualmente atrativas; fácil manuseio; interativo e com funcionalidades práticas ${ }^{20}$. Essas características divergem do resultado encontrado nas avaliações feitas, pois constatou-se que os aplicativos tinham baixa interatividade e recursos.

Outro componente importante é a funcionalidade, uma vez que a lentidão foi verificada nos aplicativos sobre sífilis. Assim sendo, os aplicativos necessitam estar disponíveis para o acesso em dispositivos móveis compatíveis com as funcionalidades, de forma a assegurar o acesso equânime entre os diversos grupos de pessoas, que podem usufruir desses dispositivos móveis e de seus benefícios ${ }^{21}$.

Salienta-se que a taxa de abandono de aplicativos geralmente é maior quando um usuário tem uma experiência ruim ${ }^{14}$. Portanto, qualquer desenvolvedor deve testar cuidadosamente os aplicativos sobre sífilis antes de colocá-los nas mãos do cliente ou usuário. Ao testar aplicativos móveis, aspectos como funcionalidade, facilidade de uso, compatibilidade, desempenho e segurança devem ser considerados, bem como a consistência e a estabilidade durante a interrupção por outros aplicativos, pela própria rede ou pelo mesmo dispositivo ${ }^{11}$.
Distintamente aos achados deste estudo, para que os aplicativos sejam incorporados de forma efetiva no cotidiano, é preciso que eles sejam atrativos visualmente, interativos e que as informações sobre saúde disponibilizadas se apresentem de modo coerente e compreensível para o usuário ${ }^{22}$.

Os aplicativos devem ser dinâmicos e ter informações consistentes para proporcionar o empoderamento dos usuários de maneira eficiente e eficaz. Diante disto, há necessidade de estudos que analisem e reflitam sobre a confiabilidade das informações disponíveis nos aplicativos sobre sífilis.

Uma revisão integrativa sobre o uso das tecnologias da informação, na educação em saúde, mostrou que é primordial mais pesquisas para investigar e analisar a efetividade das Tecnologias da Informação e Comunicação (TIC) e isso engloba a qualidade das informações que estão sendo fornecidas aos usuários ${ }^{22}$.

No presente estudo, a maioria dos aplicativos não obteve notas satisfatórias na seção de informação, o que pode comprometer a qualidade do material disponibilizado. Os estudos de validação dos aplicativos se revestem de importância, pois é a partir deles que são iniciadas etapas metódicas, que seguem determinados fluxos com critérios científicos, para tornar uma TIC validada por profissionais que dominam a temática, com o intuito de proporcionar o desenvolvimento e a disponibilização de um produto confiável para os usuários ${ }^{23}$.

Os aplicativos móveis, incluídos os que abordam sífilis, necessitam incorporar diretrizes que direcionem condutas, ações clínicas e outras práticas baseadas em evidências, porque eles são estratégias propícias de intervenção e educação em saúde das pessoas devido ao custo-efetividade, à escalabilidade e ao alto poder de alcance, quando confrontados com as intervenções convencionais ${ }^{20}$.

Para os aplicativos relacionados à saúde nas lojas virtuais de aplicativos, não são encontrados estudos de avaliação dessas tecnologias. O que está disponível ao público não foi avaliado e o que foi avaliado não está disponível ao público 6 . Diante de possíveis explicações para esse acontecimento, urge a imprescindibilidade de documentar a exploração de smartphones de uma maneira que seja facilmente acessível à comunidade científica ${ }^{24}$, embasando e fomentando pesquisas e intervenções de tecnologias móveis sobre sífilis.

Os aplicativos móveis para melhorar a saúde estão proliferando, mas, antes que os prestadores ou as organizações de serviços de saúde possam recomendar um aplicativo, são necessárias estratégias para avaliá-los. É interessante que, antes das ferramentas de tecnologia da informação e comunicação serem distribuídas, estas passem por um processo crítico, avaliativo e de aprimoramento e aperfeiçoamento ${ }^{25}$. Quando os aplicativos são validados e eficazes, eles se configuram como uma estratégia favorável para o enfrentamento de problemas de saúde pública, para o estímulo de hábitos saudáveis e para a educação das pessoas, uma vez que orientam de forma assertiva a melhor atitude a ser implementada e qual serviço de assistência à saúde deve ser buscado ${ }^{20}$. 
É imprescindível testar e avaliar exaustivamente novas abordagens promissoras, adaptando os métodos de pesquisa às necessidades dessas novas plataformas e intervenções, para que se garanta que as pessoas em risco tenham acesso ao melhor conteúdo informativo e de capacitação disponíve ${ }^{26}$. Entretanto, mais pesquisas sobre essas abordagens de tecnologias com desenhos de pesquisa rigorosos são indispensáveis para avaliar sua eficácia e custo-efetividade na promoção de comportamentos preventivos ${ }^{27}$. Ademais, é preciso ser inovador na concepção e na avaliação de futuras intervenções de saúde pública para reduzir as taxas de IST, incluindo o uso de tecnologia digital de telefonia ${ }^{28}$.

Neste estudo, foram abordados aplicativos que visam à prevenção da sífilis. Contudo, observa-se que existe um mercado amplamente inexplorado para aplicativos que melhoram as interações de usuários de dispositivos móveis com o sistema de saúde, sobretudo no que se refere à assistência à saúde sexual. Existe um interesse significativo entre os usuários em se comunicar com os profissionais de saúde e usar aplicativos para procurar serviços relacionados à saúde. O potencial desse uso de aplicativos é grande ${ }^{14}$. Nesse contexto, os programas de saúde pública precisam ser baseados em evidências e testados para garantir que os recursos selecionados sejam eficazes e adaptáveis à população em geral29.

\section{CONCLUSÃO E IMPLICAÇÕES PARA A PRÁTICA}

Este estudo forneceu uma avaliação da qualidade dos aplicativos móveis existentes nos principais sistemas operacionais sobre prevenção e controle da sífilis para a população leiga. A média de todos os aplicativos, conforme a Mobile App Rating Scale (MARS), foi de 2,8, não atingindo a pontuação mínima de aceitabilidade de 3,0 . Poucos aplicativos obtiveram pontuação média aceitável, indicando que a qualidade dos aplicativos sobre sífilis deve ser melhorada. Além disso, não foram identificadas evidências sobre a eficácia dessas intervenções. É válido salientar que somente dois aplicativos estavam disponíveis na língua portuguesa, o que mostra uma lacuna no desenvolvimento de aplicativos sobre sífilis em países lusófonos.

O uso de aplicativos móveis é uma realidade e o uso desses aplicativos requer estratégias para torná-los eficientes e eficazes no enfrentamento da sífilis. Assim, urge a necessidade de desenvolver aplicativos interativos, divertidos, customizáveis, bem orientados para o público, fáceis de manipular, com aspectos visuais adequados, bom uso de cores, tornando-os visualmente atrativos e bem descritos na loja ou dentro dos aplicativos. Com o intuito de aperfeiçoar o envolvimento entre o usuário e o aplicativo, a gamificação pode ser uma estratégia adotada.

Ressalta-se, contudo, que a pesquisa manifestou algumas limitações quanto ao instrumento utilizado para avaliar os aplicativos, uma vez que estava disponível apenas na versão inglesa e não foi validado para uso no Brasil. Além disso, foi encontrada uma limitada quantidade de aplicativos nas lojas virtuais e a não avaliação da efetividade dos mesmos. Alguns itens não puderam ser avaliados, uma vez que não foram encontrados dados suficientes sobre os aplicativos. Isso pode ter contribuído, indiretamente, para que nenhum aplicativo tenha alcançado a nota mínima de aceitabilidade na avaliação.

Portanto, torna-se essencial que novas tecnologias informacionais sejam desenvolvidas, validadas e implantadas em diversos contextos de saúde que envolvam saúde sexual e reprodutiva e, sobretudo, na prevenção de infecções sexualmente transmissíveis, como a sífilis. Sugerem-se novas pesquisas com um quantitativo maior de descritores, além do desenvolvimento de aplicativos móveis na língua portuguesa e estudos em que haja a validação dessas tecnologias sobre sífilis.

\section{FINANCIAMENTO}

Conselho Nacional de Ciência e Tecnologia (CNPq; bolsas de Iniciação científica.

\section{CONTRIBUIÇÕES DOS AUTORES}

Desenho do estudo. Nathanael de Souza Maciel. Diego da Silva Ferreira. Leilane Barbosa de Sousa

Coleta ou produção dos dados. Nathanael de Souza Maciel. Hévila Ferreira Gomes Medeiros Braga. Vitória Talya dos Santos Sousa.

Análise de dados. Nathanael de Souza Maciel. Diego da Silva Ferreira. Gutemberg dos Santos Chaves. Hévila Ferreira Gomes Medeiros Braga.

Interpretação dos resultados. Nathanael de Souza Maciel. Diego da Silva Ferreira. Gutemberg dos Santos Chaves Leilane Barbosa de Sousa.

Redação e revisão crítica do manuscrito. Nathanael de Souza Maciel. Diego da Silva Ferreira. Vitória Talya dos Santos Sousa. Hévila Ferreira Gomes Medeiros Braga. Gutemberg dos Santos Chaves. Leilane Barbosa de Sousa.

Aprovação da versão final do artigo. Nathanael de Souza Maciel. Diego da Silva Ferreira. Vitória Talya dos Santos Sousa. Hévila Ferreira Gomes Medeiros Braga. Gutemberg dos Santos Chaves. Leilane Barbosa de Sousa.

Responsabilidade por todos os aspectos do conteúdo e a integridade do artigo publicado. Nathanael de Souza Maciel. Diego da Silva Ferreira. Vitória Talya dos Santos Sousa. Hévila Ferreira Gomes Medeiros Braga. Gutemberg dos Santos Chaves. Leilane Barbosa de Sousa.

\section{EDITOR CIENTÍFICO}

Ivone Evangelista Cabral ()

\section{EDITOR ASSOCIADO}

Candida Primo Caniçali $(1)$ 


\section{REFERÊNCIAS}

1. Kostkova P. Grand challenges in digital health. Front Public Health. 2015;(3):134. http://dx.doi.org/10.3389/fpubh.2015.00134.PMid:26000272.

2. World Health Organization. MHealth: new horizons for health through mobile technologies [Internet]. Geneva:WHO; 2011 [citado 2019 abr 20]. Disponível em: http://www.who.int/goe/publications/goe_mhealth_web. pdf

3. Schnall R, Cho H, Liu J. Health Information Technology Usability Evaluation Scale (Health-ITUES) for usability assessment of mobile health technology: validation study. JMIR Mhealth Uhealth. 2018 jan;6(1):e4. http://dx.doi.org/10.2196/mhealth.8851. PMid:29305343.

4. Lucivero F, Jongsma KR. A mobile revolution for healthcare? Setting the agenda for bioethics. J Med Ethics. 2018 jun;44(10):685-9. http:// dx.doi.org/10.1136/medethics-2017-104741. PMid:29907579.

5. Bacchus LJ, Reiss K, Church K, Colombini M, Pearson E, Naved $\mathrm{R}$ et al. Using digital technology for sexual and reproductive health: are programs adequately considering risk? Glob Health Sci Pract. 2019 dez;7(4):507-14. http://dx.doi.org/10.9745/GHSP-D-19-00239. PMid:31874936.

6. Muessig KE, Pike EC, LeGrand S, Hightow-Weidman LB. Mobile phone applications for the care and prevention of hiv and other sexually transmitted diseases: a review. J Med Internet Res. 2013 jan;15(1):e1. http://dx.doi.org/10.2196/jmir.2301. PMid:23291245.

7. Rowley J, Vander Hoorn S, Korenromp E, Low N, Unemo M, AbuRaddad LJ et al. Chlamydia, gonorrhoea, trichomoniasis and syphilis: global prevalence and incidence estimates, 2016. Bull World Health Organ. 2019 jun;97(8):548-62. http://dx.doi.org/10.2471/BLT.18.228486. PMid:31384073.

8. World Health Organization. Global health sector strategy on Sexually Transmitted Infections, 2016-2021 [Internet]. WHO; 2016 [citado 2020 maio 1]. Disponível em: http://www.who.int/reproductivehealth/ publications/rtis/ghss-stis/en/

9. Ministério da Saúde (BR). Boletim epidemiológico sífilis 2019 [Internet]. Brasília: Departamento de Doenças de Condições Crônicas e Infecções Sexualmente Transmissíveis; 2019 [citado 2020 mar 26]. Disponível em: http://www.aids.gov.br/pt-br/pub/2019/boletim-epidemiologicosifilis-2019

10. Centers for Disease Control and Prevention. Sexually transmitted disease surveillance 2018 [Internet]. Atlanta: U.S. Department of Health and Human Services; 2019 [citado 2020 maio 1]. Disponível em: https:// stacks.cdc.gov/view/cdc/79370

11. Stoyanov SR, Hides L, Kavanagh DJ, Zelenko O, Tjondronegoro D, Mani M. Mobile app rating scale: a new tool for assessing the quality of health mobile apps. JMIR Mhealth Uhealth. 2015 mar;3(1):e27. http:// dx.doi.org/10.2196/mhealth.3422. PMid:25760773.

12. França VM, Carneiro NA, Medeiros BC, Danjour MF, Sousa No MV. Factors favorable to the acceptance of mobile applications: a study with Students from a public educational institution. Rev SG. 2016 mar;11(1):120-32. http://dx.doi.org/10.20985/1980-5160.2016.v11n1.1045.

13. Moon Technolabs Unip. Ltd. Apple vs Android: a comparative study 2017 [Internet]. Moon Technolabs; 2017 [citado 2020 jan 31]. Disponível em: https://www.moontechnolabs.com/apple-vs-android-comparativestudy-2017/

14. Krebs P, Duncan DT. Health app use among US mobile phone owners: a national survey. JMIR Mhealth Uhealth. 2015 nov;3(4):e101. http:// dx.doi.org/10.2196/mhealth.4924. PMid:26537656.

15. Fray JIB, McCandless MJ. The effects of using American idioms in the development of the speaking skill in L2 students. Horiz Rev Investig En Cienc Educ [Internet]. 2020; [citado 2020 maio 1];4(16):432-8. Disponível em: http://www.scielo.org.bo/scielo.php?script=sci_abstract\&pid=S261679642020000400006\&lng=en\&nrm=iso\&tlng=en
16. Molina-Recio G, Molina-Luque R, Jiménez-García AM, Ventura-Puertos PE, Hernández-Reyes A, Romero-Saldaña M. Proposal for the user-centered design approach for health apps based on successful experiences: integrative review. JMIR Mhealth Uhealth. $2020 \mathrm{abr} ; 8(4):$ e14376. http:// dx.doi.org/10.2196/14376. PMid:32319965.

17. Serrano KJ, Coa KI, Yu M, Wolff-Hughes DL, Atienza AA. Characterizing user engagement with health app data: a data mining approach. Trans Behav Med. 2017 jun;7(2):277-85. http://dx.doi.org/10.1007/s13142017-0508-y. PMid:28616846.

18. Lister C, West JH, Cannon B, Sax T, Brodegard D. Just a fad? Gamification in health and fitness apps. JMIR Serious Games. 2014 ago;2(2):e9. http://dx.doi.org/10.2196/games.3413. PMid:25654660.

19. Whiteley L, Mena L, Craker LK, Healy MG, Brown LK. Creating a theoretically grounded gaming app to increase adherence to preexposure prophylaxis: lessons from the development of the viral combat mobile phone game. JMIR Serious Games. 2019 mar;7(1):e11861. http://dx.doi.org/10.2196/11861. PMid:30916652.

20. Formagini TDB, Ervilha RR, Machado NM, Andrade BABB, Gomide HP Ronzani TM. Revisão dos aplicativos de smartphones para cessação do tabagismo disponíveis em língua portuguesa. Cad Saude Publica. 2017 Mar 9;33(2):e00178215. http://dx.doi.org/10.1590/0102-311x00178215 PMid:28300972.

21. Ferreira DP, Gomes Jr SCS. Aplicativos móveis desenvolvidos para crianças e adolescentes que vivem com doenças crônicas: uma revisão integrativa. Interface Comun Saúde Educ [Internet]. 2021; [citado 2020 maio 1];25:e200648. Disponível em: https://www.scielosp.org/article/ icse/2021.v25/e200648/

22. Pinto AC, Scopacasa LF, Bezerra LL AL, Pedrosa JV, Pinheiro PN C. Use of information and communication technologies in health education for adolescents: integrative review. Rev Enferm UFPE on line. 2017;11(2):63444. https://doi.org/10.5205/1981-8963-v11i2a11983p634-644-2017.

23. Costa PHV, Amaral NS, Polese JC, Sabino GS. Validity and reliability of movement assessment applications for smartphones: descriptive review. Rev Interdiscip Ciênc Médicas [Internet]. 2018; [citado 2020 maio 1];2(2):66-73. Disponível em: http://revista.fcmmg.br/ojs/index. php/ricm/article/view/152

24. Fiordelli M, Diviani N, Schulz PJ. Mapping mHealth research: a decade of evolution. J Med Internet Res. 2013 maio;15(5):e95. http://dx.doi. org/10.2196/jmir.2430. PMid:23697600.

25. Boceta J, Samper D, de la Torre A, Sánchez-de la Rosa R, González G. Usability, acceptability, and usefulness of an mhealth app for diagnosing and monitoring patients with breakthrough cancer pain. JMIR Cancer. 2019 abr;5(1):e10187. http://dx.doi.org/10.2196/10187. PMid:30932862.

26. Sabben G, Akelo V, Mudhune V, Ondeng'e K, Ndivo R, Stephenson $R$ et al. A smartphone game to prevent hiv among young africans: protocol for a randomized pilot study of a mobile intervention. JMIR Res Protoc. 2019 mar;8(3):e11209. http://dx.doi.org/10.2196/11209. PMid:30916661.

27. Badawy SM, Kuhns LM. Texting and mobile phone app interventions for improving adherence to preventive behavior in adolescents: a systematic review. JMIR Mhealth Uhealth. 2017 abr;5(4):e50. http:// dx.doi.org/10.2196/mhealth.6837. PMid:28428157.

28. Owens S, Kurka T, Richardson D. Public health interventions via mobile phone digital technology to reduce rates of sexually transmitted infections. J Public Health. 2019 mar;41(1):e61. http://dx.doi.org/10.1093/pubmed/ fdy052. PMid:30973959.

29. Nour M, Chen J, Allman-Farinelli M. Young adults' engagement with a self-monitoring app for vegetable intake and the impact of social media and gamification: feasibility study. JMIR Form Res. 2019 maio;3(2):e13324. http://dx.doi.org/10.2196/13324. PMid:31094322. 